\title{
Myeloid/Lymphoid Neoplasms with FGFR1 Rearrangement
}

National Cancer Institute

\section{Source}

National Cancer Institute. Myeloid/Lymphoid Neoplasms with FGFR1 Rearrangement.

NCl Thesaurus. Code C84277.

Hematologic neoplasms characterized by the rearrangement of the FGFR1 gene,

resulting in translocations with an 8p11 breakpoint. Patients present with a

myeloproliferative neoplasm, acute myeloid leukemia, lymphoblastic lymphoma/leukemia of T or B-cell lineage, or acute leukemia of mixed phenotype. 